\title{
AN OUTBREAK OF CUTANEOUS ANTHRAX IN TRIBAL AREAS OF VISAKHAPATNAM
}

\author{
G. Ajay Kumar', B. T. V. N. Raju, K. R. Harsha Vardhan'3, P. Guru Prasad4
}

${ }^{1}$ Assistant Professor, Department of Dermatology and Venereology and Leprosy, King George Hospital, Visakhapatnam. ${ }^{2}$ Assistant Professor, Department of Dermatology and Venereology and Leprosy, King George Hospital, Visakhapatnam.

3Postgraduate, Department of Dermatology and Venereology and Leprosy, King George Hospital, Visakhapatnam.

${ }_{4}^{4}$ Associate Professor, Department of Dermatology and Venereology and Leprosy, King George Hospital, Visakhapatnam.

\section{ABSTRACT}

\section{BACKGROUND}

Anthrax is a disease of herbivorous animals. Humans incidentally acquire the cutaneous disease by handling infected dead animals and their products. Sporadic cases of human anthrax have been reported from Southern India.

\section{METHODS}

Fifteen tribal men, one woman, one child from various places near Paderu presented with painless ulcers associated with vesiculation and oedema of the surrounding skin on the extremities without any constitutional symptoms. There was a history of slaughtering and consumption of a dead goat ten days - 2 weeks prior to the development of skin lesions. Three days later another 19 members came from the same area with same complaints. Clinically, cutaneous anthrax was suspected and smears, swabs, and punch biopsies were taken for culture and identification by Polymerase Chain Reaction (PCR). All the cases were treated with intravenous antibiotics followed by oral antibiotics. Appropriate health authorities were alerted and proper control measures were employed.

\section{RESULTS}

Smears from the cutaneous lesions of some patients were found to be positive for Bacillus anthracis and this was confirmed by polymerase chain reaction. All the cases responded to antibiotics.

\section{CONCLUSION}

We report thirty six cases of cutaneous anthrax in a non-endemic district, Visakhapatnam, Andhra Pradesh.

\section{KEYWORDS}

Cutaneous Anthrax, Painless Ulcer, Diseased Animals.

HOW TO CITE THIS ARTICLE: Kumar GA, Raju BTVN, P, Vardhan KRH, et al. An outbreak of cutaneous anthrax in tribal areas of visakhapatnam. J. Evolution Med. Dent. Sci. 2016;5(62):4378-4381, DOI: 10.14260/jemds/2016/999

\section{INTRODUCTION}

Anthrax is a disease of herbivorous animals caused by Bacillus anthracis and humans incidentally acquire the disease by handling infected dead animals and their products.[1][2][3][4] Cutaneous anthrax is the commonest type. The other two, inhalational and gastrointestinal anthrax are uncommon forms. Sporadic cases of cutaneous anthrax caused by biting flies have been reported.[5][6]

Anthrax is known to occur globally and it has been estimated that as many as 20,000 to $1,00,000$ human cases of anthrax occur annually generally in underdeveloped regions of the world where livestock are not vaccinated.[1][4][7] The actual incidence of anthrax in India is not known accurately mostly due to underreporting.[3][8] Many regions in India are still enzootic for animal anthrax, but it is less frequent or absent in North India and sporadic cases of human anthrax have been reported especially from South India.[9] According to a recent review of literature, there have been about 205 documented cases from India, the majority (109) of cutaneous anthrax. In the developed nations, anthrax has all, but disappeared.

Financial or Other, Competing Interest: None.

Submission 09-06-2016, Peer Review 20-07-2016,

Acceptance 26-07-2016, Published 04-08-2016.

Corresponding Author:

Dr. G. Ajay Kumar,

Assistant Professor,

Department of Dermatology and Venereology and Leprosy,

King George Hospital,

Visakhapatnam-530002.

E-mail: akgummalla@gmail.com

DOI: $10.14260 /$ jemds/2016/999
However, in India, which has got the largest population of livestock in the world, the disease is still endemic. Seventy percent of its population is living in the villages, majority are dependent on livestock for their livelihood. Moreover, a large number of people working in the tanneries and leather industries have a chance of getting infected.

The incidence of anthrax in animals and in man throughout India is not known accurately due to the fact that a large number of cases go unreported and only a fraction of human cases receive medical attention in a hospital. Cases treated "on site" in a village are hardly ever brought to the notice of the authorities. Hence, the true incidence of anthrax in man is likely to be higher than reported in the literature. Detailed information collected from three Southern Indian states, Andhra Pradesh, Karnataka, and Tamil Nadu have confirmed the endemicity of anthrax. During the last two decades, about 70 cases of human anthrax have been encountered at Christian Medical College, Vellore, of which 26 cases had cutaneous anthrax. A review of Indian literature in 1996 has found 112 cases of anthrax (71 cutaneous anthrax cases) in places other than Vellore. Infected dying animals had bleeding from various orifices and characteristic splenomegaly. In Andhra Pradesh, Chittoor, Kadapa, Guntur, Prakasam, and Nellore Districts are the known endemic areas for animal and human anthrax.[10][11] According to the information provided by the Department of Animal Husbandry, Government of Andhra Pradesh, there were 1220 animal anthrax outbreaks in Andhra Pradesh from 1991 to 2004, all in these districts. 
For the first time, thirty six cases of human cutaneous anthrax were identified in the remote tribal hamlets like Panasapottu, Goyyagunta, Vennelakota, in Araku Valley Mandal situated $140 \mathrm{~km}$ from Visakhapatnam, which is a nonendemic district of Andhra Pradesh. These cases prompted us to take up this detailed study.

\section{METHODS}

Thirty three tribal men, 2 child, 1 woman were brought with painless ulcers with surrounding vesiculation and oedema on the extremities, some on the lips, some on the back since ten days. They had no constitutional symptoms. Three weeks earlier several of their goats died of sudden illness and these people were involved in slaughtering, cooking, and eating the meat/carcass of these animals. They started developing these skin lesions 10 days after the handling of these animals.

The clinical details of all the cases are given below. On the basis of the history of contact with an infected carcass and the characteristic clinical features, a diagnosis of cutaneous anthrax was made. All patients were hospitalised and investigated. Smears and swabs taken from the vesicles, ulcers, and fluid from the surrounding oedematous region were Gram stained and cultured. Full thickness $4 \mathrm{~mm}$ punch biopsies were also taken in all the patients from the edge of the ulcers. Routine blood and biochemical investigations and chest $\mathrm{x}$-ray were done in all patients. PCR was also done to confirm the diagnosis.

All the patients were treated with intravenous ciprofloxacin $200 \mathrm{mg}$ 12-hourly along with $100 \mathrm{mg}$ doxycycline OD for the first seven days and advised to follow up by oral ciprofloxacin $500 \mathrm{mg}$ twice a day for 1 week and oral doxycycline $100 \mathrm{mg}$ BD for 6 months.

\section{RESULTS}

The direct smears of some of the suspected cases revealed thick Gram positive bacilli singly and in short chains. These findings were suggestive of Bacillus anthracis. Finally, PCR was positive for the genes encoding the Protective Antigen (PA) and also capsular region (CAP) confirming Bacillus anthracis in all patients.

Prompt clinical response to ciprofloxacin and doxycycline therapy was seen in some patients. Improvement was seen in the form of reduction of surrounding oedema within 5-7 days and eschar formation.

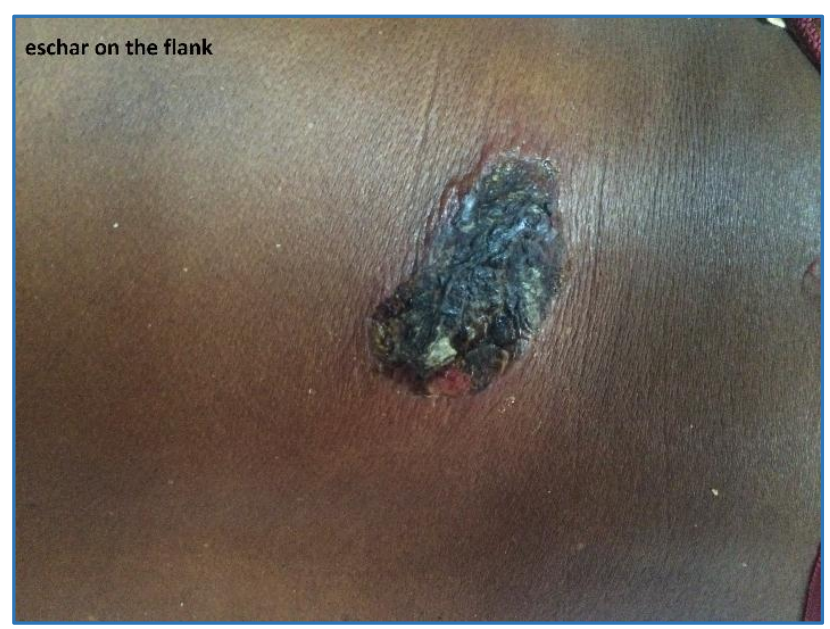

Fig. 1: Eschar on the Flank

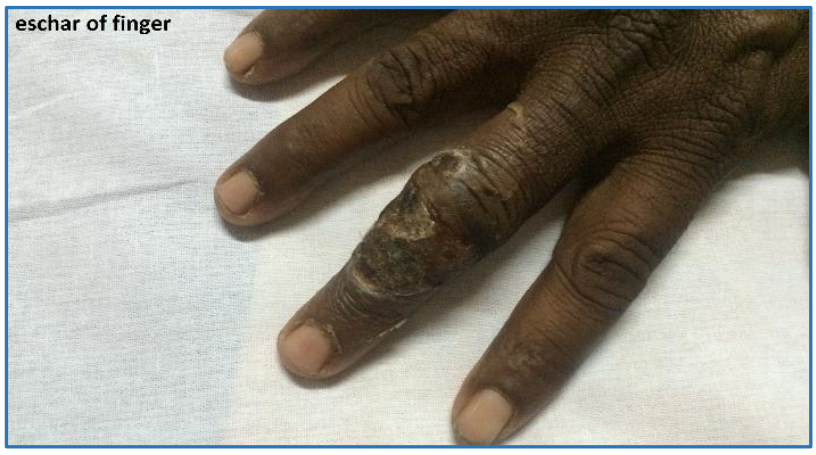

Fig. 2: Eschar of Finger

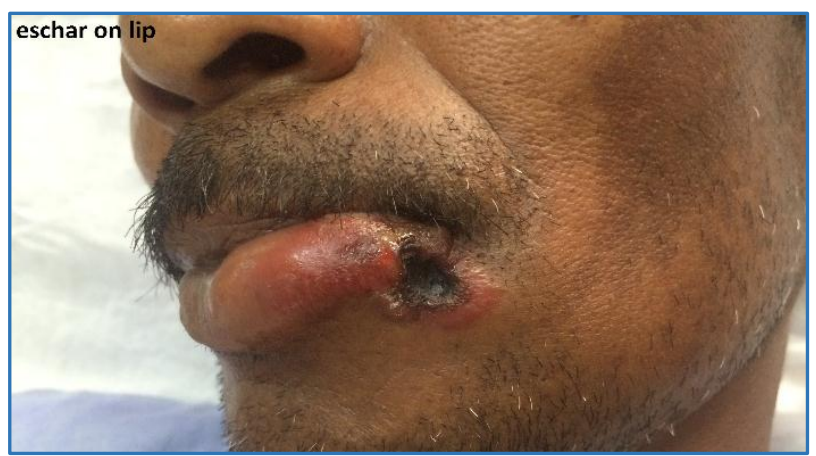

Fig. 3: Eschar on Lip

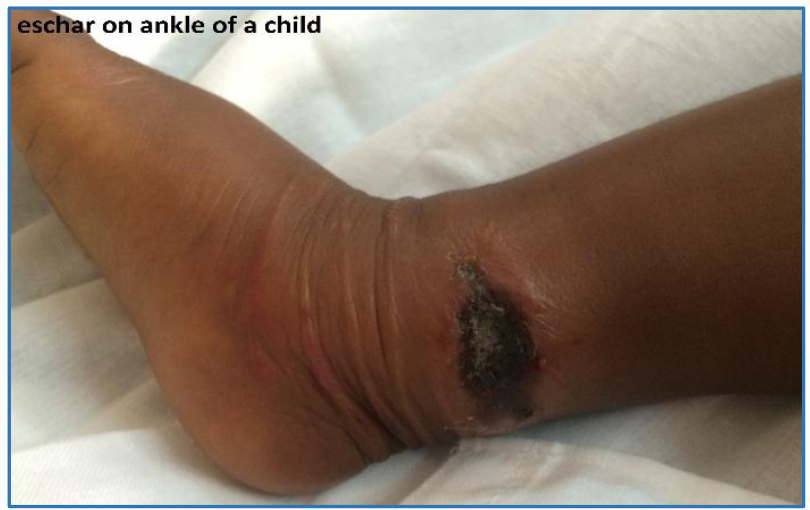

Fig. 4: Eschar on Ankle of a Child

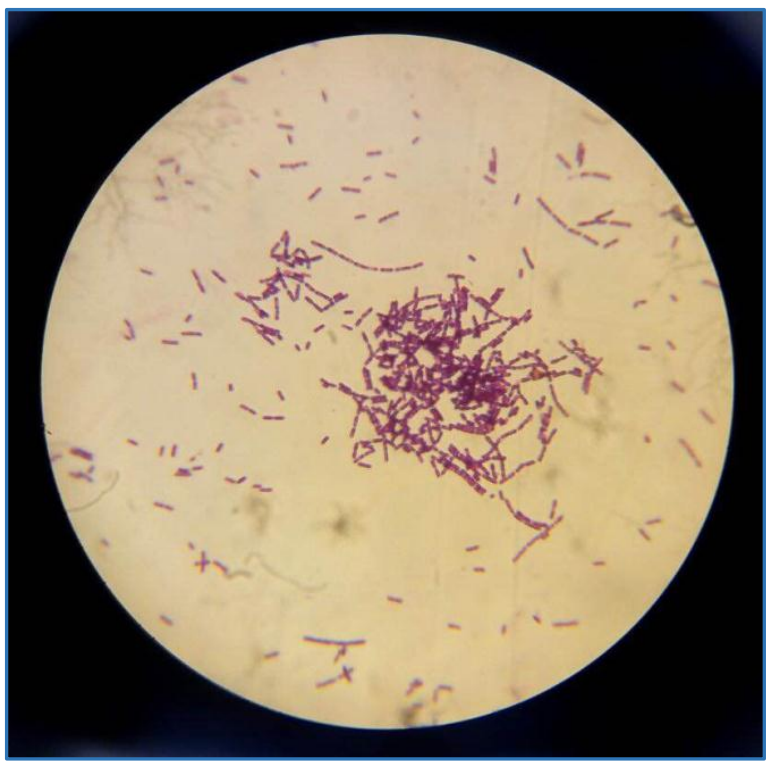

Fig. 5: Bamboo Stick Appearance 


\section{gram + bacilli in chains}

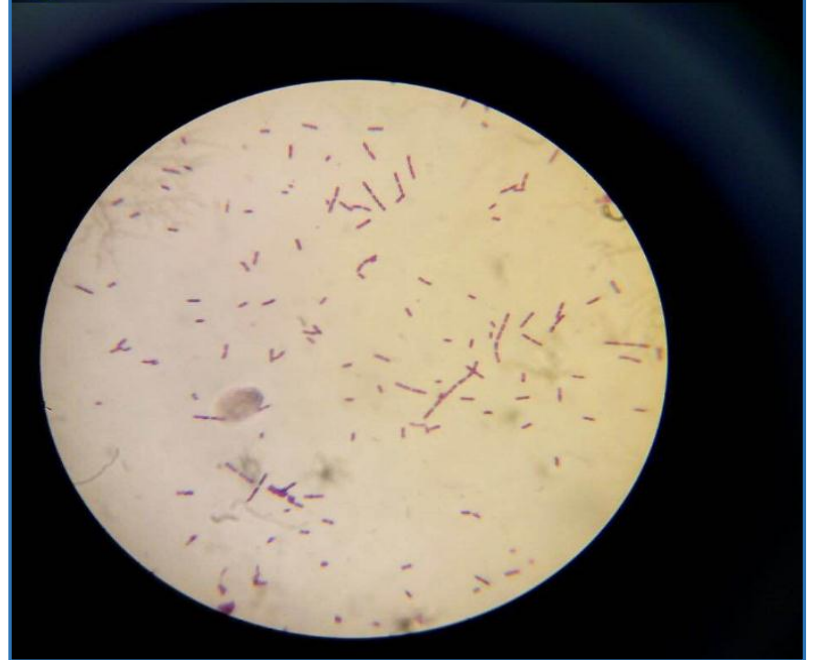

Fig. 6: Gram + Bacilli in Chains

\section{DISCUSSION}

Anthrax, a toxigenic zoonosis of herbivorous animals in which humans are an incidental host usually manifests in cutaneous form in man. Though, the disease has become rare, endemic outbreaks occur in tropical countries, parts of South America, Europe where veterinary control of livestock is marginal and environmental conditions favour an animal soil animal cycle. Many species of animals can be infected with Bacillus anthracis either natural or experimentally. There is little doubt that the most important source of the infection is domestic herbivores. These species - cattle, sheep, horses, and goats appear to be most susceptible to natural infection whereas pigs and dogs like humans can be infected only when exposed to relatively large numbers of spores. Infection in most domestic species arises chiefly by ingestion of spores. A characteristic of anthrax in animals is the inability of blood to clot as demonstrated by blood draining from the natural orifices of dead animals and skinned carcasses. This further increases soil contamination. The source of infection for animals.

Human disease is most likely to occur in endemic regions by direct contact with infected carcasses. Industrial cases may occur anywhere and reflect exposure to imported animal carcass products such as bone meal (Which is used for making glues or fertiliser), hair, or hades. Humans acquire infection by direct inoculation of spores through breaks in the skin by inhalation of spores (Generated during the early stage of cleaning contaminated goat hair), or by ingestion of contaminated meat. There may be some person to person spread in peculiar circumstances. Direct abdomen to abdomen contact was responsible for the acquisition of infection in one of our child. The other child might have acquired the infection as a result of insect. An outbreak in the Gambia was in part traced to the use of communal loofahs when bathing and in the UK and Russia shaving brushes have been a source.

During the recent outbreak of anthrax in Zimbabwe and India, there were numerous anecdotal reports of human cutaneous anthrax associated with "fly bites" B. anthracis is a large, non-motile, brick shaped, gram-positive organism (2.5 x $10 \mathrm{~mm}$ ), which occurs singly or in pairs in tissue.
The so-called classical "boxcal" appearance of long strings of bacilli is seen only in culture. On methylene blue staining, the bacterial cell is stained blue whereas the surrounding capsule is pink. This is described as the McFadyean reaction. B. anthracis has the capacity to produce heat- and dryresistant spores under adverse conditions. The organism grows well on blood agar plates. Individual colonies are stickly and stand up in stalagmite-like forms when they are lifted or touched with a bacteriologic loop. All the virulent strains of the bacillus are pathogenic for mice.

Locally, at the site of inoculation in the skin, pharynx, or intestine, low-level germination, growth, and production of exotoxins by the organisms result in extensive tissue oedema and tissue necrosis due to vascular thrombosis. In cutaneous anthrax, occasionally massive oedema may result in haemoconcentration and hypotension. In a small number of cases, systemic anthrax can lead to meningeal involvement by means of lymphatic or haematogenous spread. Cases of cutaneous anthrax have not been frequently reported in India though the disease is endemic in many parts of the country. ${ }^{[2]}$ Cutaneous anthrax accounts for $95 \%$ of all human anthrax cases[1][4][7][13]. Unlike the other forms, inhalational and gastrointestinal anthrax, it is not a life-threatening disease. Spontaneous healing occurs in $90 \%$ of cases. However, mortality in untreated cases of cutaneous anthrax is estimated to be $5-20 \%$. The characteristic clinical features of cutaneous anthrax are a painless ulcer with surrounding vesiculation along with massive oedema and eschar formation (Malignant pustule). Mild constitutional symptoms may be seen along with regional lymphadenopathy.

These lesions are seen commonly on the face, neck, and extremities. These features clinically differentiate the disease from other common infectious conditions like impetigo, cellulites, orf (Ecthyma contagiosum), milker's nodules, etc.

To diagnose cutaneous anthrax, a high index of clinical suspicion and a good history are essential. In our patients, there was history of contact with a dead animal and the characteristic lesions were seen on the extremities. The laboratory diagnosis of cutaneous anthrax depends upon recognition of the thick gram-positive bacilli in smears from the lesions.

Cultures from the skin lesions however are not useful diagnostically because the rate of positive cultures does not exceed $60-65 \%$ probably due to the prior use of antimicrobial therapy or due to the microbicidal activity of local antagonistic skin flora. Therefore, confirmation of cutaneous anthrax depends upon a positive PCR for Bacillus anthracis[1][7][8][14] even in patients who have received prior antimicrobial therapy. In some of our patients, direct smears and PCR showed Bacillus anthracis thereby confirming the diagnosis of cutaneous anthrax.

The lesion most commonly confused with cutaneous anthrax (Malignant pustule) is vaccinia, which no longer exists. Milker's nodules contracted from the teats of the cow are characterised by one or several brownish red dome shaped smooth or slightly papillomatous vegetation (Resemble pyogenic granulomas), which are generally confined to the hands and forearms. Orf (Ecthyma contagiosum) is a skin disease found usually in slaughterers or shepherds who maybe in contact with sheep suffering from ecthyma contagiosum. A virus causes it and the appearance is 
more ragged and angry looking compared to the malignant pustule of anthrax.

Moreover, Orf lacks the characteristic central eschar. Malignant pustule can be confused with a boil. However, a Gram stained smear of the exudate from the lesion usually shows the bacilli if the case is anthrax. A painful pustular eschar in a febrile patient indicates a secondary infection most often with staphylococcus or streptococcus.

\section{The Following Clinical Features if Present are Strongly Suggestive of Cutaneous Anthrax.}

1. The presence of oedema out of proportion to the size of the lesion.

2. Lack of pain during the initial phases of the infection.

3. The rarity of polymorphonuclear leucocytes from vesicular fluid on Gram stain.

The presence of typical bacilli in the Gram stained smear from the ulcer and the epidemiological proof maybe the only evidence in favour of your diagnosis of cutaneous anthrax since culture of this organism from skin lesions yields positivity in 60 to 65 percent of cases only probably due to prior antibiotic therapy of the microbicidal activity of local antagonistic skin flora. There have been reports of clinical isolates of B. anthracis that are resistant to penicillin. Because of the potential for drug-resistant strains including deliberately modified strains, antimicrobial susceptibility testing should be performed on all isolates. Several methods such as specific enzyme-linked immunosorbent assays, enzyme-linked immune-electro transfer blotting, and indirect microhemagglutination have been described for the serologic diagnosis of anthrax. The anthraxin skin test consisting of subdermal injection of a commercially produced chemical extract of an attenuated strain of B. anthracis is available for the diagnosis of acute and previous cases of anthrax. New diagnostic techniques focused on the use of the polymerase chain reaction may become useful in the near future in the clinical setting where early diagnosis is crucial. Though penicillin is the drug of choice for all forms of anthrax, betalactamase producing strains of $B$. anthracis have been reported.

Therefore, the American Academy of Dermatology recommends ciprofloxacin or doxycycline[15] and one or two additional antimicrobials for all forms of anthrax. Some of our cases responded dramatically to ciprofloxacin and doxycycline therapy and the lesions healed without scar formation. Cutaneous anthrax occurs commonly in clusters or as an outbreak in endemic areas. Recently, three outbreaks of cutaneous anthrax similar to that of ours have been reported from Mysore (1999), Midnapore (2000), and Kolar (2001).

Anthrax is a disease of public health importance and a notifiable disease. Once the diagnosis was established in our cases, the district health authorities and animal husbandry personnel were informed. Specialist teams visited the affected and the surrounding villages for door-to-door surveillance and for conducting medical camps to detect new cases. Health education camps were conducted to educate the people about the handling of dead animals and also proper disposal of carcasses by using lime. In the affected and surrounding villages, sanitary measures were taken up and the soil was decontaminated with bleaching powder. Animal husbandry authorities surveyed all the animals in these areas and found
6-8 animals suffering from anthrax (The diagnosis was established by smear and culture studies).

Dermatologists play a crucial role in the diagnosis of naturally occurring cutaneous anthrax and also in the event of bioterrorism. The purpose of this report is to create awareness about cutaneous anthrax among dermatologists.

A disease of great antiquity, anthrax occupies an important place in the history of infectious diseases because it was the first human disease to be attributed to a specific pathogen. It retains a certain fascination and notoriety partly because of the potential for use of the bacillus spores in biologic warfare.

\section{REFERENCES}

1. Thappa DM, Karthikeyan K. Anthrax: an overview within the Indian subcontinent. Int J Dermatol 2001;40(3): 216-22.

2. Hanna P. Anthrax pathogenesis and host response. Curr Trop Microbiol Immunol 1998;225:13-35.

3. Thappa DM, Karthikeyan K. Cutaneous anthrax: an Indian perspective. Indian J Dermatol Venereol Leprol 2002;68(6):316-9.

4. Morton MS, Arnold NW. Miscellaneous bacterial infections with cutaneous manifestations. In: Freedberg IM, Eisen AZ, Wolff K, et al, eds. Fitzpatrick's dermatology in general medicine. $6^{\text {th }}$ ed. New York: McGraw-Hill 2003:1918-21.

5. Turell MJ, Knudson GB. Mechanical transmission of bacillus anthracis by stable flies (Stomoxys calcitrans) and mosquitoes (Aedes aegypti and Aedes taeniorhynchus). Infect Immun 1987;55(8):1859-61.

6. Bradaric N, Punda-Polic V. Cutaneous anthrax due to penicillin-resistant bacillus anthracis transmitted by an insect bite. Lancet 1992;340(8814):306-7.

7. Wenner KA, Kenner JR. Anthrax. Dermatol Clin 2004;22(3):247-56, v.

8. Lalitha MK. Human anthrax: experience over two decades. Round table conference series number 9. New Delhi, India: Ranbaxy Science Foundation 2001:51-7.

9. Dutta KK. Emergence of anthrax as an agent of bioterrorism. Round table conference series number 9. New Delhi, India: Ranbaxy Science Foundation 2001:1120.

10. Sekhar PC, Singh RS, Sridhar MS, et al. Outbreak of human anthrax in Ramabhadrapuram Village of Chittoor District of Andhra Pradesh. Indian J Med Res 1990;91:448-52.

11. Sridhar MS, Chandrasekhar P, Singh J, et al. Cutaneous anthrax with secondary infection. Indian J Dermatol Venereol Leprol 1991;57(1):38-40

12. Lalitha MK, Kumar A. Anthrax-a continuing problem in southern India. Indian J Med Microbiol 1996;14(2):6372.

13. Taylor JP, Dimmitt DC, Ezzell JW, et al. Indigenous human cutaneous anthrax in Texas. South Med J 1993;86(1):1-4.

14. Bhatra HV. Biology and laboratory diagnosis of anthrax. Round table conference series number 9. New Delhi, India: Ranbaxy Science Foundation 2001:31-40.

15. John AC, Thomas WM, Scott AN, et al. Cutaneous anthrax management algorithm. J Am Acad Dermatol 2002;47(5):766-9. 